\title{
FUNCIONES DEL RECONOCIMIENTO EN LA PRÁCTICA EDUCATIVA
}

\author{
Functions of recognition in educational practice
}

\author{
Óscar Julián Cuesta MORENO \\ Pontificia Universidad Javeriana. Colombia. \\ ocuesta@javeriana.edu.co \\ bttps://orcid.org/0000-0001-7181-1183
}

Fecha de recepción: septiembre de 2018

Fecha de aceptación: diciembre de 2018

\section{RESUMEN}

El artículo analiza las funciones que cumple el reconocimiento en la práctica educativa, para lo cual expone la teoría sobre el reconocimiento. Desde allí, problematiza la conceptualización del reconocimiento mostrando que es una realización que supera la valoración social, puesto que es producto de un historial de vínculos intersubjetivos, para ello se usan construcciones conceptuales del psicoanálisis y la biología del conocimiento. Con este entramado conceptual, busca argumentar que el reconocimiento es un núcleo que constituye la subjetividad al establecer en el sujeto principios de interpretación que asignan relevancia a las valoraciones que hacen los otros sujetos. Siguiendo este postulado, expone el concepto de desprecio y de lucha por el reconocimiento. Finalmente, muestra que el acto educativo, como dinámica intersubjetiva, tiene como cimiento el tipo de reconocimiento que se establezca entre el docente y el estudiante, el cual de manera ideal deberá estar sostenido en el conocimiento (lo que se enseña y lo que el otro quiere aprender).

Palabras clave: reconocimiento; práctica educativa; esquemas de interpretación; vínculo; desprecio; Axel Honneth. 


\section{ABSTRACT}

The article analyzes the functions that recognition fulfills in educational practice, for which it exposes the theory about recognition. From there, the conceptualization of recognition is problematized by showing that it is an achievement that surpasses social value, since it is the product of a history of intersubjective links, for which conceptual constructions of psychoanalysis and the biology of knowledge are used. With this conceptual framework, seeks to argue that recognition is a core that constitutes subjectivity to establish in the subject principles of interpretation that assign relevance to the assessments made by other subjects. Following this postulate, exposes the concept of contempt and struggle for recognition. Finally, he argues that the educational act is based on the type of recognition that is established between the teacher and the student, which ideally should be sustained in knowledge (what is taught and what the other wants to learn).

Key words: recognition; educational practice; interpretation schemes; vinculum; contempt; Axel Honneth.

\section{INTRODUCCIÓN}

En general, el sujeto puede reafirmar el valor social de su identidad a partir del reconocimiento social que los otros le atribuyen. En ese plano, el sujeto debe su realización a una relación con los demás, pues depende de los valores que él espera que se le atribuyan en la interacción con los otros.

Esta relación puede ser resumida como la obtención de una confirmación intersubjetiva. Visto así, los juicios (valoraciones) que hacen los demás sobre el sujeto tienen una carga significativa, dado que se convierten en punto de referencia para valorarse a sí mismo.

Ahora bien, los juicios que elaboran los demás responden a esquemas de valoración. ¿Quién determina estos esquemas de valoración? Para Honneth (1997) estarían establecidos por la comunidad de valoración, que, en pocas palabras, es el núcleo de interacciones intersubjetivas donde habita el sujeto. Así, la valoración social de una persona no es la de un sujeto individualizado,

sino las de un estatus culturalmente tipificado; es un valor que, por su parte, resulta de su contribución colectiva a la realización de los objetivos sociales, según el que también se mide el valor social de cada uno de sus miembros (Honneth, 1997, p. 151).

En pocas palabras, la sociedad y sus gramáticas culturales establecen los valores o, mejor aún, los esquemas de estimación con los que un sujeto valora a otro sujeto. Y, puesto que para la realización de un sujeto es importante la valoración que hagan los otros de él, estos esquemas terminan siendo referente para los juicios que el sujeto hace de sí mismo. Diría Honneth (1997, p. 209): «los individuos se constituyen como personas solamente porque, a partir de la perspectiva aquiescente o 
alentadora de los otros, aprenden a referirse a sí mismos como seres a los que se atribuyen cualidades o capacidades positivas».

Estos esquemas de valoración afectan la construcción que el sujeto hace de sí mismo, sobre todo si les atribuye una estima exagerada, ya que puede derivar incluso en una dependencia patológica de los juicios que hacen los otros.

Sin embargo, esto no se limita a la construcción individual del self o al plano psicológico, puesto que el reconocimiento opera a nivel socioeconómico determinando la vigencia social de las profesiones, es decir, la estima que se tiene sobre determinado profesional está anclada a esquemas de valoración o aprecio que responden a gramáticas históricas y socioculturales.

En consecuencia, así como el sujeto construye juicios sobre sí mismo tomando como referente confirmaciones inter-subjetivas, del mismo modo la valoración que el profesional realiza de sí mismo está vinculada a lo que la comunidad de valoración piense de él.

En esa línea, Honneth (1997) habla de que el sujeto obtiene seguridad (autoconfianza) cuando identifica que contribuye positivamente a la reproducción de la entidad comunitaria, vale decir, a reproducir los valores que tiene la sociedad de la que hace parte.

Si la valoración del otro influye en la valoración que hace el sujeto de sí mismo, es pertinente preguntarse qué esquemas de valoración se establecen en la práctica educativa, es decir, qué papel cumple el reconocimiento en las relaciones que se configuran entre los estudiantes y el maestro. Interrogante pertinente, puesto que, en palabras de Thoilliez (2019), es más que oportuno dar una respuesta «a la necesidad de reconocimiento que el sujeto que se educa tiene» (p. 308).

Para dar cuenta de esta pregunta, en un primer momento se problematizará la idea de reconocimiento como aprobación dada por otro sujeto. Posteriormente, se recuperarán conceptos de Honneth $(1997,2011)$ para abrir esa comprensión naturalizada del reconocimiento. En tercer lugar, se mostrará cómo el reconocimiento es consecuencia de un esquema de interpretación que construye el sujeto a partir de su historial de vínculos. En seguida, se mostrará la relación que existe entre reconocimiento desprecio e invisibilización; en ese marco, se hablará de las luchas por el reconocimiento. Finalmente, se argumentará de qué forma el reconocimiento es el cimiento del acto educativo.

\section{LA PROBLEMATIZACIÓN DEL RECONOCIMIENTO MORFOLOGIZADO}

En general, la necesidad de reconocimiento aparece como el apuro que tiene el sujeto de ser distinguido por los otros sujetos. Lograr o no esta distinción produce en el sujeto confianza o desasosiego. Específicamente, cuando un sujeto se siente reconocido por el otro experimenta sentimientos de confianza y seguridad. De forma inversa, si un sujeto no se siente reconocido por el otro es embargado por el desasosiego. 
¿Qué tipo de relación está detrás de esta forma de reconocimiento? Tal como está descrito, el reconocimiento aparentemente es resultado de que el sujeto sea diferenciado, es decir, que los demás encuentren en él una identidad definida. Consecuentemente, la sensación de haber conseguido o no el reconocimiento produce, a su vez, confianza o desasosiego.

En síntesis, se puede decir que la necesidad de reconocimiento aparece como una realidad morfológica que se simplifica en una lógica lineal: el sujeto busca ser reconocido y su búsqueda tiene como consecuencia la confianza o el desasosiego.

Esta linealidad se puede abrir a partir de cuestionar el porqué de la necesidad de reconocimiento. Aparentemente, el origen de esta necesidad es resultado de una experiencia donde el sujeto es censurado por no cumplir las cualidades que esperan los otros. Esa experiencia de censura genera en él una necesidad de reconocimiento, vale decir, de que los demás distingan que sí posee cualidades.

¿Qué hay detrás de esta experiencia de censura? Se puede decir que esta experiencia de censura ocurre cuando el sujeto siente desaprobadas sus cualidades. Lo que implica, consecuentemente, que el sujeto le asigna importancia a los juicios que emiten los otros sobre sí mismo.

Precisamente, comprender por qué el sujeto le asigna importancia a los juicios que emiten los otros permite identificar las posibles contingencias que colocan al sujeto en una situación de experiencia de censura y, más aún, entender la necesidad de reconocimiento.

Honneth (2011, p. 56) piensa que la "postura de reconocimiento es expresión de la valoración del significado cualitativo que poseen otras personas o cosas para la ejecución de nuestra existencia». En la definición que hace Honneth, el término valoración cumple un papel articulador que puede ayudar a re-semantizar la idea de experiencia de censura y, específicamente, brinda elementos para comprender por qué los sujetos le asignan importancia a los juicios que los otros emiten sobre él.

En efecto, la valoración para Honneth (1997) estaría encuadrada en el orden de las relaciones de reconocimiento social, donde el sujeto puede reafirmar el valor social de su identidad. En sus palabras, «se alude a la colocación positiva frente a sí mismo, que un individuo puede aceptar cuando es reconocido como un determinado tipo de persona por los miembros de la comunidad" (p. 100). En ese plano, el sujeto debe su realización a una relación con los demás, pues depende de los valores que él espera que se le atribuyan en la interacción con los otros.

Lo anterior permite decir que la experiencia de censura estaría vinculada a que el sujeto se sienta criticado porque sus cualidades no se acoplan a los esquemas socioculturales de valoración. Como resultado de esta experiencia de censura el sujeto reacciona, entre otras cosas, buscando el reconocimiento.

Visto así, la necesidad de reconocimiento va más allá de lograr una distinción, de que los otros vean en él una identidad definida, como se había planteado en un principio, dado que esta necesidad implica, por un lado, comprender esquemas 
de valoraciones socioculturales, y por otro lado, una relación intra-subjetiva determinada por una confirmación intersubjetiva (sentirse reconocido por los otros «significa para el sujeto singular poder tomar posición positiva frente a sí mismo» [Honneth, 1997, p. 101]).

Siguiendo a Honneth (1997), la necesidad de reconocimiento existe como una exigencia de reciprocidad, en la medida que la relación de reconocimiento implica que los sujetos necesitan cierta confirmación del otro:

Si yo no reconozco al otro en la interacción como un determinado tipo de persona, tampoco puedo verme reconocido como tal tipo de persona en mis reacciones, porque a él precisamente debo concederle las cualidades y facultades en que quiero ser confirmado por él (Honneth, 1997, p. 53).

Lo anterior permitiría entender, desde otro lugar, la aparente linealidad expuesta en un comienzo: obtener reconocimiento produce confianza, no obtenerlo desasosiego. En efecto, Honneth (1997) habla de que el sujeto obtiene seguridad cuando identifica que contribuye positivamente a la reproducción de la entidad comunitaria, vale decir, a reproducir los valores que tiene la sociedad de la que hace parte.

\section{LA BúSQUEdA de RECONOCIMIENTO COMO UNA RELACIÓN MÁs ALLÁ de LA VALORACIÓN SOCIAL}

En la dinámica de valoración el sujeto espera que, en la interacción con el otro, se le atribuyan valores. Por ello se puede hablar que, de fondo, el reconocimiento está vinculado a la relación que construye el sujeto con los otros sujetos y consigo mismo. En ese orden, problematizar el concepto de relación permitiría ampliar aún más la problematización del reconocimiento.

La relación, en el marco del reconocimiento como afectación que determina la subjetividad social, está centrada en el lazo que construye el sujeto con los otros sujetos. ¿Qué tipo de lazo construye un sujeto que busca el reconocimiento?

Algunos elementos de la teoría de Melanie Klein permiten comprender la forma en que el sujeto, desde su primera infancia, va construyendo su subjetividad en lazo con los otros sujetos, especialmente, con su madre:

El mundo interno consiste en objetos, el primero de todos la madre, internalizada en varios aspectos y situaciones emocionales. Las relaciones entre estas figuras internalizadas, y entre las mismas y el yo, tienden a ser experimentadas -cuando predomina la ansiedad persecutoria- principalmente como hostiles y peligrosas; son vividas como buenas y amorosas cuando el niño es gratificado y prevalecen los sentimientos felices. Este mundo interno, que puede describirse en términos de relaciones y sucesos internos, es el producto de los propios impulsos, emociones y fantasías del niño (Klein, 1955, introducción). 
En la relación que tiene el bebe con su mamá empieza a darse un proceso que va determinando la subjetividad del niño, dado que el niño empieza una dinámica de introyectar y proyectar un mundo a partir del vínculo que establece con ella.

Se establece, pues, un vínculo con un objeto gratificante y con uno frustrante. El primero, que Klein llamó pecho bueno, está determinado por las experiencias gratificantes (ser acariciado, mimado, protegido). El segundo, llamado pecho malo, incluye las experiencias de abandono que el bebe percibe (cuando la mamá se demora en acudir a su llanto, por ejemplo). Desde la teoría del pecho bueno y pecho malo, el bebe va desarrollando un mundo fragmentado entre lo gratificante y lo frustrante, valga decir, entre lo placentero y lo que le genera displacer.

Estos elementos pueden ser traídos para comprender, desde otro marco, la búsqueda del reconocimiento como afectación que determina la subjetividad, dado que puede ser leído desde la gratificación o frustración que obtiene un sujeto al sentirse valorado por los otros sujetos.

En este caso, extrapolando los conceptos de Klein, el sujeto que busca reconocimiento construyó una relación con los otros determinada por la gratificación o frustración que da el pecho bueno o pecho malo de la valoración social. Obsérvese que los conceptos de gratificación y frustración (placer y displacer) permiten re-sementizar los de confianza y desasosiego planteados en la compresión naturalizada del reconocimiento, dado que le suman la dimensión psicoanalítica y van más allá de verlos como un resultado de la aprobación o desaprobación que hacen los otros.

En esa línea, el objeto transicional, propuesto por Donald Winnicott, es otro concepto que permite ampliar la comprensión de la relación que construye un sujeto que busca reconocimiento.

Este autor plantea que el objeto transicional es resultado de un espacio intermedio entre lo que el niño percibe como subjetivo y objetivo. En sus palabras, es una "zona intermedia entre la realidad interna del individuo y la realidad compartida del mundo que es exterior a los individuos» (Winnicott, 1972, p. 197).

La idea de objeto transicional le permite a Winnicott formular el concepto de espacio o área transicional, es decir, un área intermedia entre el niño y otra persona o el niño y la realidad. Este concepto de espacio transicional puede ser retomado para comprender la forma en que el sujeto que busca reconocimiento construye sus relaciones intersubjetivas. En efecto, un sujeto que configura una necesidad de reconocimiento podría estar atribuyendo a la valoración que hacen los demás las cualidades de un objeto transicional.

De esta manera, el sujeto que busca reconocimiento ha construido un espacio transicional donde habitan objetos transicionales que le generan gratificación. Objetos que, como se ha señalado, son al mismo tiempo objetivos y subjetivos: la valoración que hace el otro es exterior al sujeto (objetiva), pero su atribución como placentera o frustrante es subjetiva. 
Lo que dice Winnicott y Klein tiene algo en común: el niño va configurando una relación intra-subjetiva e intersubjetiva centrada en la construcción de objetos vinculados al placer o displacer. Sin embargo, ambos autores muestran de fondo que esa construcción es un proceso en el cual el sujeto tiene un lugar central, pues si bien los conceptos de pecho bueno y pecho malo y espacio transicional están asociados al vínculo del niño con su madre, el niño es el que construye el esquema de gratificación, no es un asunto que depende absolutamente de una exterioridad llamada mamá.

Precisamente, esto último permite ampliar la problematización de la relación que construye un sujeto que necesita del reconocimiento, ya que deja de ser una relación determinada por una exterioridad, más exactamente por la valoración que hacen los otros sobre las cualidades del sujeto. Así, la teoría del reconocimiento que traía Honneth necesita de una ampliación, debido a que la búsqueda de reconocimiento ya no se centra en una necesidad de confirmación intersubjetiva que brinde al sujeto seguridad sobre la valoración que él tiene de sí mismo.

\section{EL RECONOCIMIENTO COMO UNA AFECTACIÓN DETERMINADA POR LA INTERPRETACIÓN QUE EL MISMO SUJETO CONSTRUYE}

Recolocar el reconocimiento como resultado de una relación intra-subjetiva y no solamente intersubjetiva permite abordar desde otro lugar la pregunta de cuál es el tipo de lazo que construye un sujeto que busca reconocimiento. Efectivamente, el lazo que construye el sujeto que busca reconocimiento ya no sería un asunto determinado únicamente por la valoración expresada por el otro sino tendría que ver con la forma en que el propio sujeto asume esta expresión, valga decir, la forma en que la interpreta.

Para poder ampliar esta comprensión es pertinente entender el concepto de relación más allá de una conexión. De hecho, el espacio o área transicional de Winnicott da claves para observar que la relación no es una suerte de materialidad que permite el enlace entre el sujeto y otro sujeto o el sujeto y la realidad exterior, sino que la relación es un intersticio inasible en la medida que puede ser definida como un espacio de interpretaciones posibles.

En efecto, si se lee la relación simplemente como una conexión podría darse por hecho que el vínculo que se establece entre sujetos garantiza una construcción común de sentido, es decir, que lo que dice un sujeto es entendido de manera impoluta por el otro sujeto, lo que impediría cualquier ambigüedad en las relaciones humanas. Sin embargo, como esto no es así, puede verse que los vínculos entre los sujetos tienen una naturaleza menos controlada y más contingente.

Para desarrollar más la idea de que la relación es un espacio de interpretaciones posibles se traerán conceptos de dos autores: Humberto Maturana y Viktor Frankl. Si bien estos autores no hablan explícitamente sobre el reconocimiento, algunos de sus conceptos brindan claves para observar cómo el reconocimiento, más que un 
asunto de necesitar la valoración que hacen los otros para confirmar la valoración de sí mismo, depende de esquemas de interpretación que el sujeto ha construido.

Maturana (2001, p. 43) plantea que «Los seres vivos somos sistemas determinados por nuestra estructura. Nada externo a nosotros puede especificar lo que nos pasa. Cada vez que hay un encuentro, lo que nos pasa depende de nosotros». Llevado este planteamiento a un plano de la comunicación entre personas, el autor dice que cada sujeto escucha desde sí mismo, desde su propio determinismo estructural: "Lo que yo digo es una perturbación que gatilla en cada uno de ustedes un cambio estructural determinado en ustedes, y no en lo que yo digo, y, por lo tanto, no por mí, que sólo soy la contingencia histórica» (ídem).

Visto así, la relación intersubjetiva más que una conexión lineal es un vínculo de múltiples posibilidades, pues cada sujeto, de acuerdo a su estructura, decodificará el lazo que establece con el otro sujeto. Esto permite comprender que el reconocimiento no se limita a una dependencia que tiene un sujeto sobre las valoraciones que hacen los otros sujetos sobre él, dado que no es un asunto determinado taxativamente por lo que diga el otro, sino por lo que el propio sujeto interpreta de lo que dice el otro:

Si miramos con inocencia el encuentro de A y B, nos parece que A determina lo que le pasa a B, pero no es así. Más aún, a B le pasan cosas determinadas en B según su presente estructural, pero éste ha surgido de una historia de interacciones mucho más amplia que su espacio de encuentros con A. (Maturana, 2001, p. 44).

Lo que formula Maturana (2001) permite considerar una comprensión del reconocimiento no focalizada en la valoración que hacen los otros, sino en una interpretación que el sujeto hace de esa valoración. Si bien Maturana no habla de interpretación, sino de determinismo estructural, se puede decir que dicho determinismo es producto de estructuras de interpretación.

De hecho, para seguir con las palabras del mismo Maturana, estas estructuras se producen por interrelaciones recurrentes que van gatillando en el sujeto conductas congruentes. Es decir, la necesidad de reconocimiento es resultado de un proceso al interior de la subjetividad del sujeto, pero, al mismo tiempo, configurado por su historia de relaciones intersubjetivas que, como se vio en Klein y Winnicott, tiene origen en la primera infancia y, como lo muestra Honneth, depende de esquemas socioculturales de valoración que operan a nivel intra e intersubjetivo.

En esa línea, es pertinente traer el trabajo de Viktor Frankl, dado que ha mostrado como las personas sufren, no tanto por lo que les pasa, sino por lo que interpretan de eso que les pasa. Frankl sostiene que las personas atribuyen un poder condicionante a las otras personas, de tal manera que empiezan a depender de los otros, pero que, en el fondo, los individuos no sufren por lo que dicen o hacen los otros, sino por lo que la persona piensa e interpreta de lo que hizo o dijo el otro.

Frankl (1991) habla que, en última instancia, el hombre es quien se determina a sí mismo. Es decir, que no está totalmente determinado y condicionada por las 
circunstancias o por los otros sujetos, sino que al final él va a tener siempre la oportunidad de elegir qué actitud tomar frente a su vida. Dicha actitud atribuye poder al sujeto en la medida que es resultado de la lectura que él hace de sus circunstancias.

Si bien desde marcos diferentes, lo que plantea Maturana y Frankl puede articularse para mostrar cómo el reconocimiento, más que una relación de dependencia frente a la valoración que hacen los otros, es una relación de posibilidad en la medida que puede ser interpretada de distintas maneras de acuerdo a cómo asuma la valoración cada sujeto. Así, el lazo que construye un sujeto que busca el reconocimiento no tiene que centrarse en los juicios que expresan los otros sobre él, sino que necesita de un examen que permita ver por qué su subjetividad le atribuye relevancia a la valoración que hacen los otros para confirmar la valoración que él hace de sí mismo.

En ese orden, conceptos claves señalados anteriormente, como valoración y experiencia de censura, son decodificaciones que hace el propio sujeto y, como resultado de una interpretación, pueden ser alteradas si se asumen otros esquemas de lectura, valga decir siguiendo a Grüner (1995), si se utilizan otras políticas de interpretación.

Abrir de esta manera la morfología del reconocimiento le atribuye potencia al sujeto, dado que él puede definir de qué forma interpretar la valoración que hacen los otros y, de fondo, lo recoloca frente a la propia valoración que hace de sí mismo.

\section{El PAPEL de los VÍNCULOS EN LA CONSTRUCCIÓN DE LA INTERPRETACión DEL RECONOCIMIENTO}

La problematización del reconocimiento realizada hasta acá ha permitido observar que el sujeto que necesita reconocimiento no está buscando automáticamente juicios positivos sobre él expresados por otros sujetos, sino que su subjetividad atribuye relevancia a la valoración que hacen los otros porque ha construido, en su experiencia de interacción social, esquemas de interpretación que atribuyen importancia a dicha valoración.

En ese orden de ideas, es pertinente preguntarse cómo el sujeto construye estos esquemas de interpretación que llevan a sentir esa necesidad de reconocimiento. El concepto de vínculo propuesto por Pichon-Rivière (2008) brinda elementos para comprender la dinámica de dicha construcción.

Pichon-Rivière (2008, p. 22) plantea que el vínculo es «la manera particular en que un sujeto se conecta o relaciona con el otro o los otros, creando una estructura que es particular para cada caso y para cada momentom.

Al tener un carácter particular, cada sujeto establece vínculos específicos en su relación con los otros, incluso, las personas generan varios vínculos en el mismo grupo social: por ejemplo, las relaciones que establece con cada miembro de su familia no son las mismas. Además, como lo señala Pichon-Rivière (2008), estos 
vínculos tienen un momento, es decir, una temporalidad, lo que implica que su cimentación está abierta a nuevas contingencias.

La teoría del vínculo tiene una alta conexión con lo planteado por Winnicott y Klein, dado que el vínculo es una relación interna con un objeto que da como resultado "una conducta más o menos fija con el objeto» (Pichon-Rivière, 2008, p. 35). También se puede establecer vínculos con objetos externos, pero para comprender cómo el sujeto construye esquemas de interpretación es importante destacar la relación con el objeto interno, dado que en la teoría del vínculo se busca entender "la forma particular que tiene el yo de relacionarse con la imagen de un objeto colocado dentro de uno" (Pichon-Rivière, 2008, p. 36).

Esta imagen del objeto interno hace referencia al significado construido por el sujeto desde su experiencia de relación con el mundo, valga decir, la realidad exterior y los otros sujetos. Sin embargo, Pichon-Rivière indica que no se trata tanto de descubrir el objeto en sí sino el tipo de vínculo que el sujeto construyó, es decir, darse cuenta que el sujeto tiene una imagen interna y el yo establece una forma particular de relación con ella. En consecuencia, la reacción a un acontecimiento es particular a cada persona porque toca a un objeto interno propio que tiene cierto significado para cada quien.

En otras palabras, dos personas pudieron sufrir experiencias de censura el mismo día al recibir la reprobación de un profesor en el colegio. No obstante, cada uno de ellos convertirá esta experiencia en un objeto interno de manera diferente y, consecuentemente, cada vez que viva experiencias similares o tengan nuevamente relación con el profesor podrán responder de manera diferente. Todo dependerá del tipo de vínculo que construya, pero sobre todo de la forma en que cada uno va actualizando sus vínculos, pues

lo que es tomado en un momento dado por alguien que tiene una experiencia previa, va a modificar dicha experiencia y a integrarse luego de tal modo que en la siguiente experiencia la experiencia anterior es enriquecedora de la experiencia posterior (PichonRivière, 2008, p. 107).

A partir de los elementos anteriores es pertinente preguntarse, ¿qué le pasa a un sujeto que quiere generar relaciones de reconocimiento con los otros? ¿Qué vínculo interno ha construido?

No son preguntas fáciles de responder en la medida que cada vínculo es interno y particular al sujeto. De hecho, lo visible del vínculo son las conductas y maneras de ser del sujeto. Precisamente, estas conductas visibles son las que muestran ciertos rasgos de la relación interior que se tiene con el objeto. En otras palabras, para poder saber qué tipo de vínculo ha construido una persona que busca reconocimiento se tendrían que analizar las circunstancias y las personas con las que ésta deja ver esa necesidad de reconocimiento.

Desde allí, desde identificar el momento y las relaciones que le llevan a responder con esta necesidad, se podría empezar a identificar el tipo de vínculo que ha 
construido. En últimas, lo que dice una persona que busca reconocimiento de sí y de los otros deja latentes objetos inconscientes que operan en la conducta de la persona. En otras palabras, la reacción denominada necesidad de reconocimiento es particular porque afecta un objeto interno que tiene un significado particular para el sujeto.

Si las conductas y los enunciados que expresa una persona son lo visible del vínculo, sería necesario identificar cuáles son las conductas o enunciados que comparten las personas que necesitan reconocimiento, teniendo en cuenta que existen, como se vio con Honneth, diferentes formas del reconocimiento.

Siguiendo lo propuesto por Pichon-Rivière (2008), se puede afirmar que la búsqueda de reconocimiento no es una relación con el reconocimiento sino una respuesta al significado que un sujeto le atribuye al reconocimiento, significado que está determinado por vínculos internos anclados a experiencias externas. En ese orden, habrá situaciones donde una persona busque reconocimiento y otras donde no, todo depende de cómo el sujeto identifique la situación y la asocié a su historia personal (el vínculo que determina la interpretación).

Así, por ejemplo, la experiencia de censura que se puede sentir después de una evaluación se abordará como un reproche o el estudiante asignará otro significado dependiendo de su historial de vínculos y la imagen u objeto que ha internalizado. Aún más, la reacción que tenga frente a la censura (sea buscar reconocimiento, indiferencia, venganza, etc.) será resultado del significado que su vínculo le atribuye. En esa línea, una persona que al vivir la censura busca reconocimiento del otro habrá establecido un vínculo donde le asigna al otro el rol de dador de reconocimiento.

Así, frente a la pregunta de cómo el sujeto construye estos esquemas de interpretación que llevan a sentir esa necesidad de reconocimiento, se pude argumentar que estos esquemas están constituidos por vínculos, es decir, formas particulares de relacionarse con los otros dado que son producto de formas singulares de internalizar imágenes o construir objetos producto de la permanente actualización de la experiencia con el mundo y los otros sujetos. Estos vínculos determinan significados y estos, a su vez, establecen la respuesta (reacción) que va a tener la persona en las situaciones en las que se desenvuelve.

Si bien no se puede especificar que existe un vínculo universal en la persona que necesita reconocimiento, sí es posible decir que la necesidad de reconocimiento es lo visible del vínculo que ha construido el sujeto que busca tal reconocimiento. Además, también se puede argumentar que el vínculo configura un esquema organizador de la subjetividad e intersubjetividad en la medida que establece principios de interpretación y actuación del sujeto en el mundo.

De otro lado, un sujeto que brinda mucha importancia a los juicios que hacen los otros sobre él ha construido un vínculo de dependencia, puesto que sobrevalora el rol del otro en la realización de su reconocimiento a tal punto que difumina su propia auto-valoración. Incluso, se puede hablar de que genera un vínculo de un 
anonimato auto-impuesto, dado que se niega a sí mismo para atribuir una mayor importancia al otro; incluso, ese vínculo puede facilitar la dependencia a una sustancia psicoactiva.

Sin embargo, Pichon-Rivière (2008) señala que la actualización de los vínculos, sobre todo los que están concomitantemente desencadenando vivencias traumáticas, pueden ser abordados con tratamientos planificados o proyectos de sanación.

De acuerdo a esto, el vínculo de dependencia puede ser actualizado con un proyecto de sanación. Verbigracia, en las escuelas y universidades existen departamentos de bienestar u oficinas de orientación psicoafectiva que brindan herramientas a los estudiantes para actualizar sus vínculos y la forma en que interpretan los juicios que hacen los otros (sus padres de familia, compañeros de estudios y profesores).

\section{EL DESPRECIO COMO NEGACIÓN DEL RECONOCIMIENTO}

para Honneth (2011) existe una relación sustancial entre reconocimiento y desprecio. Afirma el autor de hecho que, si el reconocimiento intersubjetivo de las capacidades de una persona determina su auto-realización, de modo inverso, no recibir este sentimiento social del reconocimiento le puede llevar a vivir la experiencia del desprecio.

Honneth (2011) inicia su argumentación diciendo que los sujetos, en sus relaciones intersubjetivas, configuran unas expectativas mutuas, basadas en «encontrar reconocimiento por sus méritos sociales» (p. 137). Si estas expectativas no son logradas, se presenta una suerte de injusticia moral, pues la persona que no logre estas expectativas se sentirá afectada al no obtener el reconocimiento que ellas consideran merecido. El sentimiento de no obtener tal reconocimiento esperado es el desprecio, es decir, «las experiencias morales que los sujetos humanos tienen» en situaciones donde no logran sus expectativas de reconocimiento (ídem).

El sentimiento de desprecio, esto es, de la ausencia de reconocimiento, afecta la identidad de la persona: el desprecio va acompañado "necesariamente del sentimiento de una amenaza de la perdida de personalidad" (Honneth, 2011, p. 137). Esto se explica, básicamente, porque la construcción del self se hace, no sólo en un ejercicio intra-subjetivo, sino que tiene en cuenta las narraciones que efectúan los otros sobre mí.

La forma más activa de evidenciar el desprecio es, según Honneth (2011), la invisibilización. Esta puede ser entendida como el comportamiento que ejerce una persona frente a otra actuando como si esta no estuviera presente. Por el contrario, visibilidad sería reconocer las características relevantes de una persona.

En general, la invisibilización, o hacer desaparecer a alguien, no tiene que ver con la presencia física de la persona, "sino con la no existencia en un sentido social» (Honneth, 2011, p. 165). En efecto, «disponemos de la capacidad de demostrar 
nuestro desprecio a personas presentes mediante el hecho de comportarnos frente a ella como si no figurara físicamente en el mismo espacio» (p. 166).

Por ejemplo, se ejercerse la invisibilización en la calle cuando una persona al darse cuenta que viene un conocido se hace el distraído para no saludarlo. Desde esta lógica conceptual, la invisibilización no es un asunto perceptual o sensorial, pues las personas ven al otro, pero lo hacen invisible de manera deliberada. Este ejemplo prosaico, puede verse sin embrago en un aula de clase cuando un maestro ignora el aporte de un estudiante o no atiende su mano levantada pidiendo la palabra.

La invisibilidad, pues, implica un comportamiento expresado en gestos o enunciados que buscan expresar que el otro no está presente. ¿Qué le pasa a la persona que es invisibilizada? El sujeto que sufre la experiencia de invisibilización, que en el fondo es un desprecio o negación de su reconocimiento, sufre una experiencia desagradable al sentirse no percibido.

Para Honneth (2011) es importante establecer una claridad conceptual de este hecho. Ver a la otra persona implica una identificación elemental de ella, pues se distinguen algunas de sus características. Por ello, la visibilidad implica un fenómeno de dos niveles: en un primer momento, se identifica a la persona, es decir, se ve; en el segundo momento, de tipo figurado o determinado por consideraciones sociales, se reconoce que se está viendo a la otra persona (se hace explícito el reconocimiento). Así, cuando un sujeto deliberadamente solo identifica y suspende el segundo momento está ejerciendo el desprecio.

Este segundo momento, determinado por reglas sociales, permite a la persona vista darse cuenta que fue visibilizada, por ejemplo, al recibir un gesto, un saludo, etc. Entendiendo así visibilidad, la invisibilidad es un poco más clara: no notar esas reacciones por parte del otro. En pocas palabras, la exteriorización de reacciones determinadas socialmente, a manera de signo, son la expresión de que la presencia del sujeto fue considerada. Al no ver tales reacciones, habrá posibilidad de que éste viva la experiencia de la invisibilización.

En ese orden, hacer visible a alguien es un ejercicio que trasciende lo perceptual en la medida que se ancla a actos sociales y expresiones comunicativas. En este punto, Honneth (2011) hace una distinción entre conocimiento y reconocimiento. En el primero, se identifica, se ven las características del otro; en el segundo, se expresa alguna reacción positiva, como una suerte de apreciación. El conocimiento, además, sería una dinámica cognitiva al interior del sujeto. Por su parte, el reconocimiento sería un ejercicio público, que expresa que lo conocido es vigente, es decir, se expresa «algo» que va mucho más allá de identificarlo.

El que se siente invisible, en últimas, no lee en las expresiones del otro signos de su reconocimiento, es decir, siente que no recibe confirmación de su vigencia social. En ese orden de ideas, «el reconocimiento posee un carácter performativo, porque las expresiones que le acompañan simbolizan aquellas formas de reacción 
que serían necesarias para 'hacer justicia' a la persona reconocida" (Honneth, 2011, p. 172), como manifestación simbólica de una acción social.

Desde niños, como ya lo había mostrado Klein, se empiezan a dar estas expresiones de reconocimiento: tanto el niño reconoce en su madre al ser que le brinda cuidado, como la mamá reconoce en los gestos del niño sus necesidades. Sin embargo, estas lógicas o modos de expresión del reconocimiento o de hacer visible socialmente a alguien no son universales. Cada cultura, de acuerdo a sus tradiciones, establece modos específicos (tanto a nivel perceptual como figurado) para expresar que se ha visto al otro. De hecho, en el aula de clase se desarrollan protocolos para expresar este reconocimiento, como cuando los estudiantes se ponen de pie cuando entra el rector del instituto o cuando se quedan sentados si el que entró fue el conserje.

Ver al otro no solo es una realización biológica o sensorial, sino que es sobre todo una expresión efectuada desde un aprendizaje social, que designa cómo se establece la valoración del otro. A la par que los sujetos crecen van aprendiendo a diferenciar esa valoración, que en últimas expresa inteligibilidad.

Es pertinente señalar que hacer visible al otro expresa, en ese nivel figurado, una aprobación de la persona «O que se le concede vigencia en el papel tipificado por la sociedad que desempeña (amigo, señora de la limpieza, pasajero)» (Honneth, 2011, p. 173). Es decir, las expresiones de reconocimiento también expresan el rol social de una persona o las cualidades que ella ofrece en la reproducción social. Por ello, no es desfasado decir que el reconocimiento es un ejercicio comunicativo que garantiza la visibilidad social de las personas.

Esta naturaleza expresiva o comunicativa del reconocimiento permite a los sujetos confirmarse "mutuamente su vigencia social» y articular una aprobación del otro. La presencia de estos gestos, enunciados o, en general, signos, determinan la diferencia entre conocer y reconocer, entre identificar y atribuir valor o vigencia social. En efecto, una persona que vea en las expresiones del otro una reacción a su presencia se siente o, mejor, se auto-reconoce como reconocida socialmente.

Las expresiones de reconocimiento se pueden leer como meta acciones, dado que disponen de la acción a seguir. Por ejemplo, un saludo protocolario, como el que le puede bridar un estudiante a un profesor, configura un campo de acciones formales. Contrario a la sonrisa que brinda una madre a su hijo, que establece una disposición a la atención o el cariño.

De otro lado, el desprecio no sólo podría ser un comportamiento deliberado de no visibilizar al otro. Honneth (2007) habla de que este podría ser un resultado inconsciente, es decir, que olvida la condición inteligible del otro, producto, entre otras cosas, de «una praxis social en la que la mera observación de los demás se ha convertido tanto en un fin en sí mismo tal que toda conciencia de una relación social previa se extingue» (p. 137). En otras palabras, que la naturalización del ver al 
otro lleva a que se oblitere el segundo nivel de la visibilización, el reconocimiento, porque se cosifica la relación social (por ejemplo, cuando se automatiza el llamado a lista de los estudiantes en clase).

Para Honneth (2011) la expresión de reconocimiento dispuestas en el respeto «efectúa necesariamente la supresión de la inclinación egocéntrica en el sujeto» (p. 176). En otras palabras, cuando respeto a alguien renuncio a mis deseos egocéntricos, o tengo una disposición a dejar en paréntesis mis propios intereses:

en el sujeto que reconoce se efectúa un acto por el que queda descentrado, porque admite un valor a otro sujeto que es la fuente de pretensiones legitimas que menoscaban el amor que se tiene a sí mismo. 'Confirmación' o ‘afirmación' significan, por tanto, revestir con autoridad moral a personas a las que se trata, y en este sentido decretar sobre la propia persona el que se sepa obligada ella misma a la realización o a la omisión de determinadas clases de acciones (Honneth, 2011, p. 176).

Así, cuando un sujeto reconoce atribuye valor al otro e, incluso, autoridad moral, pues manifiesta una disposición moral de colocarse frente al otro. De hecho, cuando no hay expresión o disposición motivacional se presenta el menosprecio: no hay presencia de gestos de reconocimiento, es decir, no se le otorga valor a la persona como ser inteligible.

\section{SOBRE LA LUCHA POR EL RECONOCIMIENTO}

Se puede sintetizar que, por un lado, los sujetos pueden deliberadamente invisibilizar al otro y, de esta forma, negarle reconocimiento (aislando la experiencia de la persona para no convertirla en discurso o expresión). Y, por otro lado, que los sujetos invisibilizados pueden vivir la experiencia del desprecio, que es acompañada de sentimientos como rabia, vergüenza, indignación, entre otros.

Teniendo este marco presente, se puede pensar que los sujetos invisibilizados, los que sienten el desprecio, pueden efectuar acciones para lograr su reconocimiento. Honneth (2011) asocia estas acciones a un sentimiento de injusticia social, es decir, el sujeto percibe una desigual distribución de las posibilidades de valoración social.

Este sentimiento de injusticia por el repartimiento de las posibilidades de valoración social puede ser entendido como una distribución desequilibrada de las «oportunidades de formación cultural, reconocimiento social y trabajo que garantice la identidad (...) distribución estructuralmente desigual de bienes de vida inmateriales» (Honneth, 2011, p. 70). Esta distribución inmaterial está asociada a la material, pues en el capitalismo, por ejemplo, "las profesiones están sujetas informal e institucionalmente a un sistema hegemónico de valoración que adscribe oportunidades de respeto y grados de inteligencia» (p. 71). Así, las contingencias de ejercer $x$ o $y$ profesión determinaran las posibilidades sociales del reconocimiento, 
donde una profesión menos remunerada tenderá a sentir menos valoración y, por tal, más injusticia.

En esa línea, para Honneth (2011) el trabajo es un elemento importante para analizar "la apreciación social de una persona" (p. 141). En ese orden, el trabajo se ve como una experiencia moral vinculada al reconocimiento social. En consecuencia, "la formación individual de la identidad también depende de la apreciación social que recibe el propio trabajo dentro de la sociedad» (p. 143). Por ello, propone mirar la "revaloración o reorganización de determinados aspectos del trabajo" (ídem).

En ese orden, dado que «un miembro de la sociedad solo es integrado en ella gracias a mecanismos de reconocimiento mutuo", estos mecanismos "resultan controvertidos y, por ello, objeto de una lucha por el reconocimiento» (Honneth, 2011, p.43). Visto así, una sociedad crea las condiciones del reconocimiento y estas condiciones pueden ser evaluadas o, más puntualmente, convertirlas en objeto de puja.

Cuando un sujeto manifiesta deseo de luchar por el reconocimiento puede ser consciente de que no solo tiene menos acceso al capital, sino a la distribución cultural, a la dignidad, a la valoración por los esquemas sociales vigentes. Esta distribución desigual de las posibilidades de reconocimiento social impone una forma de vida, cabe decir un deber ser, que dificultad la auto-realización de las personas con menos acceso a estas condiciones sociales.

Sentirse fuera del entramado social del reconocimiento incentiva a una persona a protestar, pero esto no implica que tenga claro «de qué manera se debería luchar en contra de la experiencia del desprecio y la humillación» (Honneth, 2011, p. 145).

En todo caso, podría decirse que el sentimiento de injusticia y su transformación en lucha por el reconocimiento "consiste una y otra vez en expectativas relacionadas con el respeto hacia la propia dignidad, el honor o la integridad" (Honneth, 2011, p. 136). Sin embargo, se debe analizar en las luchas por el reconocimiento lo que Honneth llama procedimiento negativo, es decir, luchar por el reconocimiento cuando nos damos cuenta que estamos siendo invisibles o despreciados, como acción reactiva, pues podría dejar de lado una compresión mayor de las contingencias que determinaron el no reconcomiendo.

Colocados en el plano educativo, la puja por el reconocimiento está ligada intrínsecamente a la formación de culturas democráticas (Honneth, 2013), al desarrollo de prácticas altruistas y de servicio que promuevan, al mismo tiempo, respeto y autonomía (García, Rovira y Casares, 2018) y la reducción de situaciones de discriminación en los ambientes escolares y la materialización de la inclusión en escenarios educativos (Echeita et al., 2014).

Incluso, las luchas por el reconocimiento devienen en estudiantes que entran en puja con los mecanismos de valoración, pero también de los maestros que cada vez más deben recurrir a diversas estrategias para que sus estudiantes vean en ellos sujetos de saber y poder captar su atención o las luchas de diferentes colectivos y sindicatos que batallan para reivindicar la profesión del docente que, desde luego, pasa por mejorar sus salarios y prestaciones sociales. 


\section{CONCLUSIONES: EL RECONOCIMIENTO COMO CIMIENTO DEL ACTO EDUCATIVO}

El reconocimiento es un núcleo que constituye la subjetividad al establecer en el sujeto principios de interpretación que asignan relevancia a las valoraciones que hacen los otros sujetos. Esta relevancia radica en que el sujeto considera que la valoración intersubjetiva es preeminente en la confirmación de su auto-valoración. Los principios de interpretación son construidos desde historiales de vínculos que fijan significado a las expresiones de visibilidad que hacen los otros en el marco de una comunidad de valoración. Cada comunidad establece esquemas de valoración que garanticen su reproducción, en consecuencia, la valoración de un sujeto evalúa el aporte de dicho sujeto en la reproducción de la comunidad.

El reconocimiento tiene un núcleo subjetivo muy fuerte, pero, al mismo tiempo, tiene un vínculo social muy particular, dado que es otro el que reconoce. Así, el reconocimiento es una estructura de interpretación a nivel individual que determina la decodificación a nivel social. La síntesis de estos dos niveles configura un tercer nivel, el nivel simbólico del reconocimiento: el cuerpo y las cualidades del sujeto representan y conmemoran un sentido anclado a los esquemas de significado de la comunidad, de allí que el reconocimiento sea un proceso comunicativo.

Se puede ver que el reconocimiento como construcción del sujeto es un proceso simultáneo a nivel psicológico, social, cultural y existencial. Es psicológico porque involucra la construcción interior de esquemas de sentido que determinan creencias, actitudes y conductas individuales. Es social en la medida que la naturaleza del reconocimiento es inevitablemente inter-subjetiva pues por más autónoma que sea su realización, el reconocimiento se efectúa siempre en el encuentro con el otro; lo importante es señalar que un vínculo dependiente sobrevalorará la importancia del otro en el proceso. Es cultural dado que los esquemas de valoración del reconocimiento son resultado de negociaciones de significado que se adquieren y actualizan mediante gramáticas de saberes históricos y particulares a cada grupo humano.

El encuentro de estas dimensiones afecta la realización del sujeto y su reconocimiento, de allí que se pueda plantear que la conjunción de lo psicológico, social y cultural permite la realización de la existencia contingente del reconocimiento. En efecto, el reconocimiento tendría existencia contingente porque estas dimensiones no se conjugan de la misma manera en todos los sujetos, sino que se da de forma particular, lo que explica que algunos tengan necesidad de obtenerlo o dependan ansiosamente de la valoración que haga el otro sobre él.

De esta manera las condiciones de existencia del sujeto determinan la naturaleza del reconocimiento o, más exactamente, de la forma en que el sujeto lo asume y realiza su existencia con el reconocimiento como hilo de sentido de su subjetividad. De allí que, retomando a Frankl (1991), el sujeto sea dueño de la interpretación que asuma sobre su necesidad de reconocimiento y tenga potestad de la forma en que 
lo organiza en sus actitudes y conductas. Igualmente, por ello se puede alterar dicha interpretación a través de la re-actualización de los vínculos (Pichon-Rivière, 2008).

En ese orden, al ser el acto educativo un evento de encuentro entre sujetos, el reconocimiento es un catalizador del vínculo pedagógico. La formación que un sujeto quiere ejercer sobre el otro, el efecto en la subjetividad que busca lograr el docente en el estudiante, está condensado en una experiencia previa: el mutuo reconocimiento de los sujetos. Este reconocimiento pasa por conceder al otro un lugar y advertir sus cualidades, por lo que en la práctica educativa operan esquemas de valoración.

Consecuentemente, el acto educativo no puede ser disgregado de las esferas del reconocimiento planteadas por Honneth (1997), la del amor, la jurídica y la solidaridad, así como de sus respectivas realizaciones en el sujeto: autoconfianza, el autorespeto y la autoestima.

El ejercicio de reconocimiento como experiencia que sostiene al acto educativo no es siempre ideal o neutro. En efecto, en el opera también el reconocimiento reificado (Honneth, 2007), es decir, cuando se naturalizan o dan por dadas las cualidades del sujeto desconociendo la posibilidad que tiene éste de modificar sus prácticas y creencias. Por ejemplo, cuando un estudiante tiene fama de vago y pasa al siguiente año el otro profesor ya tiene un perfil condicionado por el arrastre de ese prestigio. Igualmente, en el reconocimiento del evento pedagógico se puede buscar el moldeamiento de la subjetividad del otro a través de discursos y prácticas, como lo es la apología condicionante, que destaca las cualidades de un sujeto para perfilar a los otros sujetos.

Esto implica que para promover la valoración social en el aula de clase «el profesor debe mantener una actitud de compromiso práctico con la realidad, como único medio efectivo de captar toda la complejidad de las formas de reificación y menosprecio que pueden presentarse en su contexto de actuación concreto" (Thoilliez, 2019, p. 308).

De otro lado, la pedagogía y la didáctica muchas veces obvian o naturalizan la dinámica de reconocimiento en la práctica educativa, pero es condición de su realización: la intención de formar a alguien pasa por reconocer el estado ontológico en el que el sujeto se encuentra y querer llevarlo a otro (o idealmente brindarle las condiciones para que éste llegue a otro estadio). En todo caso, la relación docenteestudiante de las instituciones de formación modernas pasan por un reconocimiento, ya sea reconocer en el otro carencias (la idea de adolecente, por ejemplo) o potencialidades (el estudiante como potencia contenida).

La práctica educativa como un encuentro intersubjetivo donde los sujetos se reafirman mutuamente establece una disposición de acuerdo al vínculo que se construye cotidianamente. En la escuela y la universidad este vínculo tiene la naturaleza de una jerarquía concedida: creo que usted sabe, por eso vengo a aprender 
de usted. Creo que usted quiere aprender, por eso me dispongo a enseñarle. Esto es un esquema ideal del proceso (Cuesta, 2017). Sin embargo, se dé o no de esta forma, siempre hay la configuración de un vínculo intersubjetivo en el proceso de formación que está determinado por el reconocimiento mutuamente establecido. Así, un estudiante que no vea con respeto a su docente ha configurado un esquema axiológico sobre el otro que determina la naturaleza del vínculo pedagógico: no le pone cuidado, le gasta bromas, indispone a sus compañeros contra el maestro, etc.

Desde esta lógica, el reconocimiento sería un factor importante en la dinámica pedagógica, toda vez que la reputación del docente (es muy bueno o es muy severo) o de los estudiantes (son un grupo difícil o son un grupo genial) tiene incidencia en el vínculo intersubjetivo que se establece en el acto educativo. En consecuencia, el reconocimiento tiene la capacidad de determinar la forma en que el proceso de enseñanza se organiza, ya que los elementos relacionales de la didáctica son catalizados por la comunicación y los condicionantes socioculturales, que a su vez tienen en el reconocimiento un principio fundador puesto que configura la densidad del tejido social. En pocas palabras, el reconocimiento puede ser un factor que estimule o cohíba la disposición didáctica de los sujetos que intervienen el acto educativo.

El reconocimiento que sirve de tejido previo al acto educativo está urdido por una relación mediada por el saber. En efecto, el saber es sustrato del vínculo que el docente estable con sus estudiantes. Básicamente, en el acto educativo se da un proceso de mutua confirmación como sujetos de saber. El sujeto-docente configura su ontología como enseñante de acuerdo a la naturaleza de la conexión que mantiene con el saber disciplinar y el saber didáctico-pedagógico. El sujetoestudiante configura su ontología como aprendiz de acuerdo a las características que tiene su deseo de saber. (Cuando el sujeto-docente estudia e investiga expresa deseo de saber, es decir, se coloca como sujeto-estudiante).

Estos vínculos con el saber, que brindan rasgos a los sujetos, estipulan el reconocimiento previo al acto educativo, materializando lo que Hegel llamó determinaciones reflejas, es decir, cuando un sujeto se estable como tal por el reconocimiento que hace el otro de él y viceversa. En este caso, el docente es tal porque hay unos sujetos estudiantes que lo reconoce como docente y viceversa.

Por otro lado, la relación que establece el docente con el saber, tanto disciplinar (su materia) como pedagógico (saber enseñarlo, evaluarlo, motivarlo), es un elemento axiológico que determina la valoración que hacen los estudiantes y colegas sobre tal profesor. Un buen docente no es tal porque tiene un título de doctor o publica en revistas indexadas (que puede ser un síntoma poroso de que sabe de su disciplina) sino porque sabe y sabe compartirlo con otro. Sin embargo, los instrumentos que evalúan al docente (criterios oficiales de valoración) no necesariamente dan cuenta de la relación que guarda el sujeto-docente con el conocimiento, de 
hecho, la obliteran o dan por dado que está manifiesta en un título, su producción o experiencia (Cuesta, 2018).

Los cambios en los criterios de valoración social del docente han logrado encubrir la relación que el docente guarda con el saber, a tal punto que se afirma continuamente que el docente es un acompañante (como el esclavo en la antigua Grecia) en un camino que ya está predefinido: el currículo y sus contenidos.

A contrapelo, el campo pedagógico-didáctico discute sobre qué sociedad se quiere y qué tipo de sujeto es necesario formar para alcanzarla (lo que hace de la pedagogía algo intrínsecamente político). En consecuencia, un docente que se coloque en disposición de reflexión pedagógica podrá cuestionar la reproducción axiológica del reconocimiento, es decir, las estrategias del orden para naturalizar cualidades que deben tener los sujetos. En efecto, un docente que no cuestione los discursos y prácticas que se colocan como hegemónicas empieza a verlas como necesarias, familiares, sin advertir que son construcciones intencionales y contingentes, es decir, que podrían ser de otra manera.

De hecho, una reflexión sobre la teoría y luchas por reconocimiento no podría ignorar que la educación parece oscilar en dos puntos: la formación de ciudadanos o la disposición de individuos para el mercado, donde el primero manifiesta, diría Honneth (en entrevista con Hernàndez I Dobon, Herzog, y Martins, 2017), una preparación de la voluntad democrática y el segundo una capacitación laboral capitalista. Ahora bien, la dinámica de reconocimiento en el mundo contemporáneo no pude entenderse sin la generación de conductas democráticas (Honneth, 2013).

\section{REFERENCIAS BIBLIOGRÁFICAS}

Echeita, G., Muñoz, Y., Sandoval, M. y Simón, C. (2014). Reflexionando en voz alta sobre el sentido y algunos saberes proporcionados por la investigación en el ámbito de la educación inclusiva. Revista latinoamericana de educación inclusiva, 8(2), 25-48.

Cuesta, O. (2017). Formación de maestros, campo pedagógico y deseo de saber. Horizontes Pedagógicos, 19 (2), 77-84.

Cuesta, O. (2018). Reconocimiento social del docente universitario: subjetividad agobiada, puja por el prestigio académico y reivindicación del acto educativo. El Ágora USB, 18(1), 4-71.

Frankl, V. (1991). El hombre en busca de sentido. Barcelona: Herder.

García, X., Rovira, J. y Casares, M. (2018). Reconocimiento y don en la educación social. Edetania, 53, 45-60.

Grüner, E. (1995). Michel Foucault: una política de la interpretación. Buenos Aires: El cielo por asalto.

Hernàndez i Dobon, F. J., Herzog, B. y Martins, M. R. (2017). La Educación y la Teoría del Reconocimiento: entrevista a Axel Honneth. Educação \& Realidade, 42(1), 395-406. http://dx.doi.org/10.1590/2175-623670297

Honneth, A. (1997). Las luchas por el reconocimiento. Barcelona: Crítica. 
Honneth, A. (2007). Reificación. Buenos Aires: Katz.

Honneth, A. (2011). La sociedad del desprecio. Madrid: Trotta.

Honneth, A. (2013). La educación y el espacio público democrático. Un capítulo descuidado en la Filosofía política. Isegoría, 49, 377-395.

Klein, M. (1955). Sobre la identificación. Recuperado de: http://www.elortiba.org/old/mklein/ melanie.Klein.6.30.htm (Consultado el 27/11/2018).

Maturana, H. (2001). Emociones y lenguaje en educación y política. S.L.: Psikolibro. Recuperado de: http://www.matriztica.cl/wp-content/uploads/Emociones-y-Lenguaje-en-Educaciony-Politica.pdf (Consultado el 27/11/2018).

Pichon-Rivière, E. (2008). Teoría del vínculo. Buenos Aires: Nueva Visión.

Thoilliez, B. (2019). Vindicación de la escuela como espacio para el desarrollo de experiencias democráticas: aproximación conceptual a las prácticas morales de reconocimiento y respeto. Educación XX1, 22(1), 295-314.

Winnicott, D. (1972). Realidad y juego. Buenos Aires: Galerna. 